\title{
Transitioning Nuclear Fuel Cycles with Uncertain Fast Reactor Costs
}

\section{U. B. PHATHANAPIROM ${ }^{1}$, E. A. SCHNEIDER}

The University of Texas at Austin, Department of Mechanical Engineering, 204 East Dean

Keeton Street, Austin, TX 78712

${ }^{1}$ E-mail: bphathanapirom@utexas.edu

TOTAL PAGES: 35

NUMBER OF FIGURES: 6

NUMBER OF TABLES: 7

(C) 2016. This manuscript version is made available under the Elsevier user license http://www.elsevier.com/open-access/userlicense/1.0/ 


\begin{abstract}
This paper applies a novel decision-making methodology to a case study involving choices leading to the transition from the current once-through light water reactor fuel cycle to one relying on continuous recycle of plutonium and minor actinides in fast reactors in the face of uncertain fast reactor capital costs. Unique to this work is a multi-stage treatment of a range of plausible trajectories for the evolution of fast reactor capital costs over time, characterized by first-of-a-kind penalties as well as time- and unit-based learning. The methodology explicitly incorporates uncertainties in key parameters into the decision-making process by constructing a stochastic model and embedding uncertainties as bifurcations in the decision-tree. "Hedging" strategies are found by applying a choice criterion to select courses of action which mitigate "regrets". These regrets are calculated by evaluating the performance of all possible transition strategies for every feasible outcome of the uncertain parameter. The hedging strategies are those that preserve the most flexibility for adjusting the fuel cycle strategy in response to new information as uncertainties are resolved.
\end{abstract}

Keywords:

Decision-making under uncertainty; fuel cycle transition analysis 
Transitioning Nuclear Fuel Cycles with Uncertain Fast Reactor Costs

U. B. PHATHANAPIROM ${ }^{1}$, E. A. SCHNEIDER

The University of Texas at Austin, Department of Mechanical Engineering, 204 East Dean

Keeton Street, Austin, TX 78712

${ }^{1}$ E-mail: bphathanapirom@utexas.edu

\section{INTRODUCTION}

Time-dependent analyses of the nuclear economy, for instance to assess transitions between nuclear fuel cycles, often confront uncertainties by implementing a scenario-based approach in which uncertain variables are parametrically varied. However, large uncertainties in the cost, performance, and even availability of technologies associated with advanced nuclear fuel cycles are present and will likely remain unresolved for decades (Shropshire et al., 2009). Due to these uncertainties, a single optimal strategy for transitioning from one nuclear fuel cycle to another does not exist. Strong transition strategies should be flexible, enabling reasonable outcomes to be attained once these uncertainties are resolved.

This work applies a novel methodology, originally presented in (Phathanapirom and Schneider, 2015), to a case study involving transition from the current once-through light water reactor (LWR) fuel cycle to one relying on continuous recycle of plutonium and minor actinides in fast reactors (FRs) where the capital cost of FRs is uncertain and evolves through time. The methodology hinges on a decision tree analysis approach for incorporating uncertainties as future bifurcations in a single, coherent model, based on the principles of decision-making under uncertainty. Unique to this article is a multi-stage treatment, including first-of-a-kind (FOAK) penalties, of plausible trajectories for the evolution of FR capital costs over time.

\section{BACKGROUND}

Modern decision theory provides a systematic approach to choosing between alternative courses of action under conditions of imperfect knowledge, which may refer to either 
information or foresight. The work presented here deals with decision-making under imperfect information, or uncertainty, where one or more decision-relevant parameters have many possible outcomes or end-states. This is termed the no-data decision problem, which consists of four components: (1) the available actions that can be taken, (2) the states of nature (or end-states) which may occur, (3) the consequences of each combination of action and state of nature (known as a state-act pair), and (4) a choice criterion by which the decision maker solves the final problem of choice.

Several techniques have been proposed to address the no-data problem (Gorenstin et al., 1993). Of these techniques, scenarios analysis has been the most pervasive for handling uncertainty in the nuclear fuel cycle. The scenarios approach finds an optimal plan for $N$ possible scenarios, obtaining a set of $N$ solutions. This approach assumes agents have perfect information about the state of nature that will prevail, and no systematic method is available for consolidating the plans to incorporate the uncertainties that are actually present. Instead, this work presents a methodology for handling uncertainties in the nuclear fuel cycle derived from concepts utilized in stochastic optimization. Here, uncertainties are explicitly embedded as future bifurcations with assigned probabilities within a single model. Using this model, a single strategy is found whose performance is optimal "on the average" for all scenarios.

The Dynamic Systems Analysis Report for Nuclear Fuel Recycle (DSARR) utilizes the scenarios approach to examine systems costs, uranium resource impacts, and waste management impacts involved in transitioning fuel cycles from the current once-through (Dixon et al., 2008). The scenarios examined include once-through; single tier recycling in FRs only; and two tier recycling, first in LWRs, and then in FRs. One uncertainty considered by the DSARR report is the overnight capital cost of LWRs and FRs. The total cost of electricity is calculated for various values of these parameters and contrasted. Using a stochastic optimization approach, a hedging strategy (typically a partial closing of the fuel cycle, see (Phathanapirom and Schneider, 2015)) would be chosen that allowed the greatest amount of flexibility until the uncertainty could be resolved. The strategy, using total cost of electricity as a metric, would minimize the additional 
costs accrued by following the hedging strategy compared with the costs if any feasible outcome for the uncertain parameter occurred. Then, a full closure of the fuel cycle, or abandonment of the transition, would occur, depending on the outcome of the LWR and FR capital costs.

\section{METHODOLOGY}

The transition scenario examined here is described and cast as a no-data decision problem in Section 3.1 Section 3.2 presents a methodology for selecting the optimal transition strategies under perfect information, as well as a general method, based on principles of stochastic programming, for selection of optimal hedging strategies. Section 3.3 briefly describes the VEGAS fuel cycle simulator, the chosen analysis platform, and documents all input simulation data (Schneider and Phathanapirom, 2015).

\subsection{Reference transition scenario}

The scenario considered here involves transitioning from the current once-through LWR fuel cycle towards continuous recycle of plutonium and minor actinides in low conversion ratio FRs, depicted in Fig. 1. LWR uranium oxide (UOX) used fuel (UF) from existing LWRs is separated into fission products (FPs) for storage and subsequent disposal as HLW, uranium for reuse or storage, and transuranic (TRU) elements. TRU elements are then burned in FRs, producing electricity while achieving partial TRU destruction. FR used fuel is subsequently separated into the same three streams: U, FPs, and TRU. Separated TRU is then recycled and again burned in FRs. This closed fuel cycle utilizing FRs with a conversion ratio of 0.5 is aimed at minimizing the existing TRU inventory, similar to Evaluation Group 24 found in the Department of Energy's Fuel Cycle Options study (Wigeland et al., 2014). FR reprocessing capacity is assumed wholly adequate and may correspond to builds of co-located reprocessing and fuel fabrication facilities, demonstrated previously at the Idaho Integral Fast Reactor facility adjacent to EBR-II (Shropshire et al., 2009). Legacy LWR SNF ${ }^{\mathrm{i}}$ is assumed to go directly to disposal, and has no bearing on the decision made. This is consistent with current policy following a technical review by Oak Ridge National Laboratory ${ }^{\text {ii }}$. 
One key source of uncertainty regarding transition to the closed fuel cycle depicted in Fig. 1 is the capital cost of FRs. Given this uncertainty, the potential benefits of the transition are unclear. If FR costs are high relative to LWRs, the transition could be delayed or even abandoned at the expense of continued usage of natural resources and repository capacity. Alternatively, if FR costs are low relative to LWRs, it would likely be optimal to pursue an aggressive schedule for closing the fuel cycle.

Simulating transition strategies between once-through and continuous FR recycle while incorporating the uncertainty in FR capital costs gives rise to a no-data decision problem, depicted in Fig. 2. Table I accompanies Fig. 2 and provides a timeline of the information available to agents during each stage of the transition. The decision time period extends from 2015 to 2100, though simulations are run through 2160 (an additional lifetime of the longest operating facility) to ensure that liability costs are accounted for ${ }^{\mathrm{iii}}$. The possible FR capital cost end-states are given in Table II. The lowest, medium, and highest end-states are taken from the low, nominal, and high estimates from the Advanced Fuel Cycle Cost Basis report (Shropshire et al., 2009) and represent engineering estimates. The low and high end-states are taken as equidistant between the medium and lowest, and medium and highest end-states respectively. A FOAK penalty is applied to the first 8 FRs built, according to the Cost Estimating Guidelines for Generation IV Nuclear Energy Systems (EMWG, 2007). According to (EMWG, 2007), direct construction costs are assumed to decline by 6 percent with each doubling of capacity due to "learning elasticity", up to $8 \mathrm{GW}_{\mathrm{e}}$ of installed capacity ${ }^{\mathrm{iv}}$. For instance, a FOAK facility with direct construction costs of $\$ 1,000 \mathrm{M}$ can expect a decline to $\$ 940 \mathrm{M}$ for the second facility. Averaged over the first 8 facilities, this equates to a FOAK penalty of approximately 8 percent over the $\mathrm{n}^{\text {th }}$-of-a-kind (NOAK) facility.

FR construction and operation can only go forward if a supply of separated TRU is available. As described further in Section 3.3, the fuel cycle simulation platform used for this study will only build FRs if they are supplied with TRU over their full 60-year lifetime. Subject to that constraint, it will build as many FRs as possible. Therefore, the degree of transition to a 
symbiotic LWR-FR fleet is directly controlled by the availability of LWR used fuel reprocessing capacity (FR used fuel reprocessing is not limited).

In 2030, the option to build a pilot 500 tIHM/yr LWR UF reprocessing facility is available. Alternatively, agents may choose to wait to build the pilot facility until 2045 . If the pilot facility is built, FOAK FRs are built with low, medium, or high construction costs plus the FOAK penalty, see Table II. These intermediate states are accessible from chance nodes $\mathrm{C}_{1}$ and $\mathrm{C}_{3}$ in Fig. 2, and the outcome at these chance nodes filters the possible end-states that may occur. For instance, if the low cost occurred at $\mathrm{C}_{1}$, then only the lowest, low or medium end-states may occur following a positive build decision at the next decision node. The parameters $\mathrm{C}_{1}$ and $\mathrm{C}_{3}$ in Table II govern the probability that each of these FR capital cost intermediate states are realized if FRs begin to be built after 2030 and after 2045, respectively. Each intermediate state is equally likely to occur if the pilot facility is built in 2030 (see Table I, $\mathrm{C}_{1}$ probability), however, waiting until 2045 skews the probability and favors the low and medium end-states occurring (see Table $\mathrm{I}, \mathrm{C}_{3}$ probability). The option to wait ${ }^{\mathrm{v}}$ is interpreted as allowing for further research and development of FR technology, increasing the probability that FR capital costs will decline. The assumption here is that as time passes, the likelihood of technological innovations improves (Balcer et al., 1984), (Farzin et al., 1998).

Following the construction of the pilot reprocessing facility, a larger 3,000 tIHM/yr reprocessing facility may be built, or the transition may be abandoned and new FR construction ceases. These NOAK FRs will be built with the low, medium, or high cost (depending on the outcome at chance node $\mathrm{C}_{1}$ or $\mathrm{C}_{3}$ ). When deciding to construct the first large reprocessing facility, the agent will already know the outcome of $\mathrm{C}_{1}$ or $\mathrm{C}_{3}$ and hence the cost of all FRs to be built for the next 15 years. But it is reasonable to expect that FR capital costs will subsequently continue to evolve. FR costs may increase over time, for instance due to regulatory evolution as was the case for LWR costs during the 1960s-1980s (Hultman et al., 2007). They may also decline as the technology continues to evolve and time- and unit-based learning ${ }^{\mathrm{vi}}$ continues. Therefore, the FR capital cost may change again 15 years after the first large reprocessing 
facility is built. Probabilities $\mathrm{C}_{2}$ and $\mathrm{C}_{4}$ govern the likelihood that FR capital costs will change at this time. By default, these probabilities are defined so that there is a $1 / 3$ probability that FR capital costs will remain the same, $1 / 3$ that they will increase to the next cost level shown in Table II, and 1/3 that they will decrease to the previous level. The outcome at $\mathrm{C}_{1}$ and $\mathrm{C}_{3}$ filters the possible end-states that may occur; for instance, if the low cost occurred at $\mathrm{C}_{1}$, then only the lowest, low, or medium end-states may occur following a positive build decision at the next decision node.

At the time that this final information regarding FR capital costs becomes known, the agent may choose to build a final 3,000 tIHM/yr reprocessing facility. This option is available in 2060 (2075) if the pilot facility was built in 2030 (2045), and another larger facility in 2045 (2060). The agent has full knowledge of the capital cost of all FRs built subsequent to this date. Therefore, a decision not to build the third reprocessing facility would indicate that FR capital costs from 2060 forward have become unacceptably high.

\subsection{Hedging Strategy Selection}

For simplicity, selection of hedging strategies is explained for the two-stage problem, where there is one state of nature for stage one and a defined number of possible states of nature for stage two, which an agent must hedge against. A generalized method for selecting optimal hedging strategies for the $N$-stage problem is given in (Phathanapirom and Schneider, 2015). The key difference in the $N$-stage problem is there are hedging strategies associated with every stage except for the final one which follows the complete resolution of the uncertainty. At intermediate stages, the uncertainty is partially resolved and the agent gains some information about the state of nature that will ultimately prevail. Thus there will be a hedging strategy associated with each adjusted expectation for the end-state based on the partial information obtained. Hedging strategy selection for the full reference transition scenario depicted in Fig. 2 may be viewed as a nested two-stage problem in which the regret for following a hedging strategy at stage $N-1$ is minimized based on the best outcome for following another hedging strategy at stage $N$. The regret may be considered a measure of the suboptimality of a strategy, or the opportunity loss 
through the agent choosing a hedging strategy that does not exactly match the perfect information strategy.

The example two-stage problem in Fig. 3 is a subset of the reference transition scenario, depicted in Fig. 2, where it is assumed that the option to build the $500 \mathrm{tIHM} / \mathrm{yr}$ pilot reprocessing facility has been undertaken. Let $h=1,2$ index over the hedging strategies available before the uncertainty is resolved (in Fig. 3, these correspond to the first decision node's option to build the 3,000 tIHM/yr reprocessing facility and the option to stop building FRs). Note that if the decision is to not build an additional reprocessing facility and to stop building FRs, currently existing FRs continue operating until the end of their lifetime utilizing the reprocessing capacity already installed. Then, let $p=1,2$ index over the perfect information strategies available after the uncertainty is resolved (in Fig. 3, these correspond to the second decision node's option to build another 3,000 tIHM/yr reprocessing facility and the option to stop building FRs). All strategies may then be identified by $j_{h, p}$, where $j_{h, p}=(h-1) * 2^{1}+p$ (Table II enumerates $j_{h, p}$ for all $h$ and $p$ ). Finally, let $i=1,2,3$ index over the possible end-states shown in Fig. 3.

For each end-state $i$, an optimal strategy $j_{i}^{*}$ is found from among the possibilities $j_{h, p}=1$, 2... 4 according to Eq. 1:

$$
j_{i}^{*}=\underset{h, p}{\operatorname{argmin}} \sum_{t=1}^{T} c_{t}^{i, j_{h, p}} \cdot E_{t}
$$

where $E_{t}$ is the electricity produced in year $t$, and $c_{t}^{i, j}$ be the calculated levelized cost of electricity (LCOE) for the $i^{\text {th }}$ end-state, the $j^{\text {th }}$ expansion strategy, and the $t^{\text {th }}$ year, $t=1,2 \ldots T$. Here, the optimal transition strategy is chosen as the strategy which minimizes the energyintegrated LCOE over the decision time period. These optimal transition strategies $j_{i=1,2,3}^{*}$ correspond to the decisions that would follow if an agent had perfect information about which end-state would prevail. 
Given these optimal transition strategies, it is possible to identify the optimal hedging strategy $h^{*}$ under the limits of imperfect information. First, the regret for following the hedging strategy $h$ given that the $i^{\text {th }}$ end-state occurred, $r_{h}^{i}$, is given in Eq. 2:

$$
r_{h}^{i}=\underset{p}{\operatorname{argmin}}\left(\sum_{t=1}^{T} c_{t}^{i, j_{h, p}} \cdot E_{t}-\sum_{t=1}^{T} c_{t}^{i, j_{i}^{*}} \cdot E_{t}\right) .
$$

According to Eq. 2, $r_{h}^{i}$ is taken as the minimum difference in the value of the objective function for strategy $j_{h}$ (over options $p$ ) and the perfect information strategy $j_{i}^{*}$. Minimizing over the options $p$ takes into account that an agent, who followed the hedging strategy $h$ prior to the resolution date, may pick the strategy $p$ that minimizes the regret $r_{h}^{i}$ once it is known that the $i^{\text {th }}$ end-state occurred. Given the choice of the objective function, the regret may take on various meanings; in the context of this work, regrets represent foregone savings, and the maximum regret incurred may place an upper bound on the value of perfect information.

Finally, given these regrets, a choice criterion is applied to find the optimal hedging strategy $h^{*}$. Here, the choice criteria used aim to minimize the maximum and expected regret, according to Eq. 3 and 4, respectively.

$$
\begin{gathered}
h^{*}=\underset{h}{\operatorname{argmin}}\left\{\max _{i} r_{h}^{i}\right\}, \\
h^{*}=\underset{h}{\operatorname{argmin}}\left\{\sum_{i=1}^{I} P_{i} \cdot r_{h}^{i}\right\} .
\end{gathered}
$$

In Eq. $4, P_{i}$ is the probability of the $i^{\text {th }}$ end-state occurring. The optimal hedging strategy selected according to Eq. 3 is independent of the probability distribution on cost outcomes as it only hedges against the worse-case outcome, and may be considered more "robust", whereas the strategy selected according to Eq. 4 is heavily dependent on the probability distribution of the cost outcomes as the expected regret is calculated using the probability distribution on the endstates. 


\subsection{Fuel Cycle Simulations}

VEGAS, a dynamic simulation tool for the nuclear economy, is chosen here as the analysis platform since it offers a physical and economic model of the nuclear fuel cycle while offering a reduced runtime over more detailed fuel cycle systems tools. Full documentation of the methodology utilized by VEGAS, particularly its mass balance and reactor deployment algorithms and LCOE calculation methodology, is provided in (Schneider and Phathanapirom, 2015).

The reference transition scenario input parameters are given in Table III, with parameters characterizing the two reactor types deployable in the scenario given in Table IV. Each VEGAS simulation begins in 2015 with a $100 \mathrm{GW}_{\mathrm{e}}$ fleet of advanced LWRs and a nuclear electricity demand growth rate of 1.25 percent per year ${ }^{\text {vii }}$. The VEGAS simulations are carried out through 2160 (an additional lifetime of the longest operating facility) to ensure liability costs are accounted for. However, only the 2015 to 2100 period is used for the decision analysis.

Prior to 2030, VEGAS meets electricity demand targets by constructing advanced pressurized water reactors (APWRs). After 2030, VEGAS prioritizes the construction of FRs (if there is supporting LWR used fuel reprocessing capacity). VEGAS attempts to build FRs to satisfy demand, but if a FR cannot be supplied fuel - because there is insufficient TRU available, or insufficient reprocessing capacity to recycle the TRU from used fuel - VEGAS's unique rollback feature is implemented. The roll-back feature moves the simulation clock back in time to the construction date of the newest FR and replaces that reactor with an APWR. In this way, VEGAS ensures that the installed reprocessing capacity is utilized to the fullest extent possible, and the maximum number of FRs that may be supported by the reprocessing infrastructure are being built. In this way, a simulation is fully specified by its reprocessing capacity expansions profile. For more details on VEGAS's roll-back algorithm, see (Schneider and Phathanapirom, 2015).

Each VEGAS simulation calculates the annual LCOE in cents per kWh of electricity produced. The LCOE is the cost at which electricity must be generated in order to attain break even and consists of three components: front end fuel cycle charges, back end fuel cycle charges, 
and reactor charges. Default unit costs are assigned to each distinct fuel cycle process in VEGAS, though fuel cycle unit costs may also be customized for each reactor technology. Unit costs are given in dollars per unit of mass throughput, typically $\mathrm{kg} \mathrm{U}$, IHM, or SWU. An independent unit cost is defined for separated actinide (TRU) storage and disposal. Reactor cost inputs are broken into capital costs (dollars) and annual operations and maintenance costs (dollars per year).

All fuel cycle technology cost data is provided in Table IV and is obtained from (Shropshire et al., 2009) unless otherwise noted. Source in Table IV indicates the table look-up for (Shropshire et al., 2009). Table F2/D2-7 of (Shropshire et al., 2009) gives a reference unit cost of $\$ 6,000 / \mathrm{kg}$ IHM for integral electrochemical processing of FR metal fuel, including FR fuel fabrication. Little cost data is available in which the two processes are separated. Unit costs for the two processes are obtained by applying a 2:3 ratio between fuel fabrication $(\$ 2,400 / \mathrm{kg}$ IHM) and reprocessing (\$3,600/kg IHM), obtained from (Platt, 2007), summarized in Table F2/D2-4 of (Shropshire et al., 2009).

The capital cost of FRs is uncertain, and the effects of this uncertainty on decisionmaking are the focus of this work. Therefore, five possible end-state cost outcomes (described in Section 3.1) are selected and their respective probabilities given in Table I. For LWRs, an overnight capital cost of $\$ 5000 / \mathrm{kW}_{\mathrm{e}}$ is used; this is the upper-bound value from (Shropshire et al., 2009) ${ }^{\text {viii }}$. Updates to two major studies, the 2003 Future of Nuclear Power prepared by MIT and the 2004 University of Chicago Study, since the publication of (Shropshire et al., 2009) indicate that LWRs may be more costly than previously anticipated. In fact, both doubled their projected reactor capital overnight cost since the 2003-04 timeframe. The previous reference value of $\$ 2000 / \mathrm{kW}_{\mathrm{e}}$ for LWRs was updated to $\$ 4210 / \mathrm{kW}_{\mathrm{e}}$ and $\$ 4000 / \mathrm{kW}_{\mathrm{e}}$ in $($ Rosner et al., 2011) and (Deutch and Forsberg, 2009), respectively.

\section{RESULTS}

This section presents results from the reference transition scenario described in Section 3.1. In addition, a sensitivity study was performed which focuses on varying the $C_{1,3}$ and $C_{2,4}$ 
parameters in Fig. 2, which govern the probability of each FR capital cost intermediate state and end-state occur, respectively.

\subsection{Reference Transition Scenario}

The first step toward finding the hedging strategies is to follow the traditional scenarios approach to find the optimal strategies for each FR cost outcome assuming that perfect information regarding that outcome was available beforehand. Table VII gives the perfect information strategies for all possible end-states. Note that the perfect information strategy is dependent on both the "intermediate" state (the partial information provided following the build of the pilot reprocessing facility) and the end-state. Thus the lowest-cost strategy depends on both the cost of the first few FRs built and the degree of learning that subsequently takes place, as quantified by the end-state.

All perfect information strategies associated with the lowest, low or medium end-states build the pilot facility as soon as possible rather than waiting in 2030 . While the medium endstate has lower capital costs for FRs as compared to LWRs, the LWR-FR fuel cycle remains more costly than the once-through fuel cycle due to reprocessing costs outstripping extra used fuel disposal costs. Hence, when the medium or high final end-states occur, additional reprocessing facilities and FR builds are not pursued beyond 2060. In this case, enough reprocessing and FR capacity has been built by 2060 to avoid the need to dispose any LWR used fuel in the repository prior to 2100 and cost savings from avoiding repository capacity usage and disposal costs outweighing the additional reprocessing costs. However, as long as the FR cost end-state is at least medium, it becomes uneconomic to replace as many LWRs with FRs as possible and it is more effective to abandon the transition. Hence, the integrated costs for both the medium and high final end-state are identical, since the agent has foregone building additional reprocessing capacity and abandons further FR builds.

Once the perfect information strategies are determined, the hedging strategies may be found. Fig. 4 gives the optimal hedging strategies for the lowest maximum and lowest expected regret choice criteria, along with the regrets accumulated by the hedging strategies selected and 
the end-states realized. Boxes in Fig. 4 signify opportunities to build reprocessing facilities; white boxes indicate a decision not to build a facility while green boxes indicate a decision to build. It is important to note that a white box in Fig. 4 not only indicates foregoing the option to build a reprocessing facility, but also ceasing to build FRs from that point onward (existing FRs continue to operate for their entire lifetime).

Looking at the top panel of Fig. 4Error! Reference source not found., it is evident that when the lowest maximum regret choice criterion is applied the optimal hedging strategy in 2030 is to delay the start of the transition to FRs until the next opportunity in 2045. This delay modifies the probabilities of the cost outcomes in favor of increased likelihood for lower FR costs, but delaying comes at the expense of building more LWRs and facing a larger inventory of used fuel needing reprocessing should the transition prove desirable. By 2060, when the next opportunity to build a reprocessing facility arises, the agent knows the intermediate stage cost but not yet the final cost. There are now three hedging strategies, one for each intermediate cost. The upper panel of Fig. 4 shows that if the intermediate cost is low or medium, under the lowest maximum regret choice criterion the agent finds it worthwhile to build the next reprocessing facility. Under high costs, no further facilities are built. Recall that the decision to build the last reprocessing facility is made in the presence of full information: the cost of building FRs from then onward is known.

The hedging strategies for the lowest expected regret choice criterion are shown in the lower panel of Fig. 4. The hedging strategies are generally the same as was the case for the lowest maximum regret choice criterion, except that the agent decides not to wait when the first opportunity to build a reprocessing facility arises in 2030. Recall that the lowest maximum regret choice criterion seeks to minimize the sub-optimality of the worst case outcome. As seen in the results from the lowest expected regret choice criterion, by building immediately in 2030 the regrets incurred when the high intermediate state occurs is $\$ 64.3$ billion. If the first build is delayed until 2045 , though, the regret is $\$ 57.4$ billion. Neither case is optimal from the standpoint of perfect information, as the best perfect information strategy when intermediate 
stage FR costs are high is always to build zero reprocessing facilities. Nonetheless, the hedging strategies call for small reprocessing facilities to be built in 2030 (lowest expected regret choice criterion) or 2045 (lowest maximum regret choice criterion) because there is sufficient chance that FR costs will turn out to be medium or lower. But under the lowest maximum regret choice criterion it is advantageous to delay because the worst case outcome - of high FR costs - is less suboptimal under the delayed start. The lowest maximum regret choice criterion should be considered the more conservative of the two choice criteria used here, as regrets incurred when this choice criterion is applied are less than under the lowest expected regret choice criterion where relatively high regrets are tolerated for some outcomes as long as the probability-weighted average regret is minimized.

When the lowest regret choice criterion is applied, the optimal hedging strategy identified is to build the pilot facility in 2030 instead of waiting. This behavior reflects that $2 / 3$ of the perfect information strategies suggest building the pilot facility in 2030. In both cases, if the first few FRs are built with the high cost, the decision should always be to abandon the transition to the synergistic LWR-FR fuel cycle and cease new FR builds.

\subsection{Sensitivity Study}

The reference transition scenario may be viewed as an $N$-stage no-data decision problem (see Section 3.2) where $N$ is 3, characterized by the information available during the time period $^{\mathrm{ix}}$. This section addresses the sensitivity of the hedging strategy outcomes to the probabilities assigned to various cost trajectories. These probabilities only affect the hedging strategies arrived at under the lowest expected regret choice criterion. The lowest maximum regret choice criterion, which only hedges against worst-case outcomes no matter their likelihood, is unaffected by the probabilities.

Fig. 5 presents the expected regret for the stage 1 hedging strategies as a function of the probability of the high intermediate FR capital cost state occurring, $P(H)$, (altering the $\mathrm{C}_{1}$ and $\mathrm{C}_{3}$ parameters in Fig. 2). The low and medium intermediate states are then treated as equally likely, such that their probabilities of arising, $P(L)$ and $P(M)$, are given in Eq. 5 . 


$$
P(M)=P(L)=\frac{1-P(H)}{2}
$$

There are three possible stage $N-2$ hedging strategies: build the pilot facility in 2030 (Y in Fig. 5), wait and build it in 2045 (W-Y), and don't built it at all (N-N). The expected regret for building the pilot facility in 2030 is the smallest up to $P(H)$ of about 0.65 . Once the likelihood of the high intermediate capital cost exceeds 0.65 , it becomes optimal to never build the pilot facility and entirely forego closing the fuel cycle: the chance that FR capital costs will prove to be low enough to make the transition economically favorable has become too small. Since $P(H)$ was $1 / 3$ in the reference transition scenario, the optimal hedging strategy for stage $N-2$ was to build the available pilot reprocessing facility, as reflected in Fig. 4.

Once stage 2 is reached and the intermediate FR cost information is gained, there is one optimal hedging strategy for each intermediate information outcome. Fig. 6 presents the expected regret for the stage 2 hedging strategies (given the options taken in stage 1) as a function of the probability of the high end-state occurring given that the medium intermediate state occurred, $P(H \mid M)$, (altering the $\mathrm{C}_{2}$ and $\mathrm{C}_{4}$ parameters in Fig. 2). Again, the low and medium end-states are treated as equally likely, and their probabilities of arising are found as previously given in Eq. 5. There are five possible stage 2 hedging strategies: build the pilot facility in 2030, then build a second larger facility in 2045 (Y-Y) or don't build it (Y-N); wait and build the pilot facility in 2045, then build a second larger facility in 2060 (W-Y-Y) or don't build it (W-Y-N); and abandon the transition $(\mathrm{N}-\mathrm{N})$.

The expected regret for the Y-Y hedging strategy is always 0 , and so it is always the best hedging strategy to follow regardless of the probability of the high end-state occurring. This occurs since Y-Y are the first two decisions in the perfect information strategy for each of the possible end-states resulting from the medium intermediate information outcome. Table VII shows that if the low end-state occurs, the perfect information strategy is Y-Y-Y (build all three facilities), while if the medium or high states occur, the strategy is instead Y-Y-N. 
Since $P(H \mid M)$ was $1 / 3$ in the reference transition scenario, the optimal hedging strategy for stage $N-2$ was to build the available larger reprocessing facility, as reflected in Fig. 4 . However, if the option to build the pilot facility in 2030 were removed for any reason, the optimal hedging strategy in 2045 would be to build the pilot facility at that time if $P(H)$ is up to about 0.58 , see Fig. 5 . Then, given these decisions, the optimal hedging strategy in stage $N-1$ becomes W-Y-Y for $P(H \mid M)$ up to about 0.85 .

\section{CONCLUSIONS}

Previous approaches to fuel cycle transition analyses have assumed that decision-makers would be able to correctly guess the value of uncertain parameters in the future. In contrast, the methodology used here, originally presented in (Phathanapirom and Schneider, 2015), systematically devises hedging strategies for fuel cycle transition analysis. These hedging strategies represent a "middle-of-the-road" approach to decision-making, preserving options until uncertainties are lessened or resolved.

A case study involving a transition from the current once-through LWR fuel cycle to one relying on continuous recycle in FRs is presented. The transition is subjected to uncertainty in the capital cost of FRs. In all cases, at least a partial transition to a closed fuel cycle is suggested

through the building of a pilot LWR used fuel reprocessing facility. Depending on information of FR capital costs resultant from this build, the transition is either abandoned or accelerated.

While not explicitly calculated here, the framework allows for the calculation of the option value of waiting. This real options analysis interpretation of the framework may be the subject of future work.

\section{ACKNOLWEDGEMENTS}

This research is being performed using funding received from the DOE Office of Nuclear Energy's Nuclear Energy University Programs.

\section{References}

1. Balcer, Y., \& Lippman, S. A. (1984). Technological expectations and adoption of improved technology. Journal of Economic Theory, 34(2), 292-318. 
2. Deutch, J., \& Forsberg, C. (2009). Update to the MIT 2003 Future of Nuclear Power. Massachusetts Institute of Technology.

3. Dixon, B., Halsey, B., Kim, S., Matthern, G., Piet, S., \& Shropshire, D. (2008). Dynamic systems analysis report for nuclear fuel recycle. Idaho National Laboratory, doi, 10, 963737.

4. Economic Modeling Working Group (2007). Cost estimating guidelines for Generation IV nuclear energy systems. Gen IV International Forum, GIF/EMWG/2007/004

5. Farzin, Y. H., Huisman, K. J., \& Kort, P. M. (1998). Optimal timing of technology adoption. Journal of Economic Dynamics and Control, 22(5), 779-799.

6. Gorenstin, B. G., Campodónico, N. M., Costa, J. P., \& Pereira, M. V. F. (1993).

Power system expansion planning under uncertainty. Power Systems, IEEE Transactions on, 8(1), 129-136.

7. Hultman, N. E., Koomey, J. G., \& Kammen, D. M. (2007). What history can teach us about the future costs of US nuclear power. Environmental science \& technology, 41(7), 2087-2094.

8. OECD Nuclear Energy Agency (2010) "Uranium 2009: Resources, Production and Demand," OECD Nuclear Energy Agency, Paris.

9. Phathanapirom, U. B., \& Schneider, E. A. (2015). Nuclear fuel cycle transition analysis under uncertainty. Nuclear Science and Engineering, (accepted 8/2015).

10. Platt, (2007). Combining LWRs, Fast Reactors and ADS Called Best P\&T Option. Platt's Nuclear Future, pp. 3-4.

11. Rosner, R., Goldberg, S., Hezir, J. S., \& Davis, E. M. (2011). Analysis of GW-Scale Overnight Capital Costs. EPIC, University of Chicago, Technical Paper Nov.

12. Schneider, E. A., \& Phathanapirom, U. B. (2015). VEGAS: A fuel cycle simulation and preconditioner tool with restricted material balances. Nuclear Technology, (accepted 8/2015).

13. Shropshire, D. E., Williams, K. A., Boore, W. B., Smith, J. D., Dixon, B. W., DunzikGougar, M., ... \& Schneider, E. (2007). Advanced fuel cycle cost basis. Idaho National Laboratory, INL/EXT-07-12107.

14. Wagner, J. C., Peterson, J. L., Mueller, D., Gehin, J. C., Worrall, A., Taiwo, T., ... \& Halsey, W. (2012). Categorization of Used Nuclear Fuel Inventory in Support of a Comprehensive National Nuclear Fuel Cycle Strategy (No. ORNL/TM-2012/308). Oak Ridge National Laboratory (ORNL).

15. Wigeland, R., Taiwo, T. A., Ludewig, H., Todosow, M., Halsey, W., Gehin, J., ... \& Oakley, B. (2014). Nuclear Fuel Cycle Evaluation and Screening-Final Report. USA: US DOE Fuel Cycle Technologies, 8. 


\section{TABLE CAPTIONS}

Table I. Timeline for information provided during reference transition scenario.

Table II. Possible fast reactor capital costs.

Table III. Enumeration of strategies for the two-stage decision problem.

Table IV. Summary of reference transition scenario input parameters.

Table V. Reactor input parameters.

Table VI. Unit cost data for reference scenario.

Table VII. Perfect information strategies for end-state histories. 
Table I. Timeline for information provided during reference transition scenario.

\begin{tabular}{c|c||c|c||c|c||c|c}
$\begin{array}{c}\text { Build in } \\
2030 ?\end{array}$ & FR cost 2030-45 & \multicolumn{1}{c}{$\begin{array}{c}\text { FR cost } \\
2045-60\end{array}$} & \multicolumn{1}{c}{ Build in 2045? } & $\begin{array}{c}\text { FR cost 2060- } \\
\text { onward }\end{array}$ & \multicolumn{1}{c}{$\begin{array}{c}\text { Build in } \\
2060 ?\end{array}$} & Build in 2075? \\
\hline $\mathrm{Y}$ & $\begin{array}{c}\text { Intermediate } \\
\text { state } i+\text { FOAK }\end{array}$ & $\begin{array}{c}\text { Intermediate } \\
\text { state } i\end{array}$ & $\mathrm{Y}$ & $\begin{array}{c}\text { End-state } i-1, \\
i \text { or } i+1\end{array}$ & $\mathrm{Y}$ & N/A \\
\hline $\begin{array}{c}\text { Build in } \\
2030 ?\end{array}$ & FR cost 2030-45 & $\begin{array}{c}\text { Build in } \\
2045 ?\end{array}$ & FR cost 2045-60 & FR cost 2060-75 & $\begin{array}{c}\text { Build in } \\
2075 ?\end{array}$ & $\begin{array}{c}\text { FR cost 2075- } \\
\text { onward }\end{array}$ & $\begin{array}{c}\text { Build in } \\
2075 ?\end{array}$ \\
\hline $\mathrm{W}$ & $\mathrm{N} / \mathrm{A}$ & $\mathrm{Y}$ & $\begin{array}{c}\text { Intermediate } \\
\text { state } i+\text { FOAK }\end{array}$ & $\begin{array}{c}\text { Intermediate } \\
\text { state } i\end{array}$ & $\mathrm{Y}$ & $\begin{array}{c}\text { End-state } i-1, \\
i \text { or } i+1\end{array}$ & \begin{tabular}{c}
$\mathrm{Y}$ \\
\hline
\end{tabular}
\end{tabular}

"Y" - build reprocessing facility; "N" - don't build reprocessing facility; "W" - wait; "N/A" - not applicable 
Table II. Possible fast reactor capital costs

\begin{tabular}{lcccc} 
End-state & NOAK Cost $\left(\mathbf{\$} / \mathbf{k} \mathbf{W}_{\mathbf{e}}\right)$ & FOAK Penalty $\left(\mathbf{\$} / \mathbf{k} \mathbf{W}_{\mathbf{e}}\right)$ & $\mathbf{C}_{\mathbf{1}}$ Probability & $\mathbf{C}_{\mathbf{3}}$ Probability \\
\hline Lowest & 3000 & N/A & 0 & 0 \\
Low & 3600 & 3900 & $1 / 3$ & $2 / 5$ \\
Medium & 4200 & 4500 & $1 / 3$ & $2 / 5$ \\
High & 5600 & 6000 & $1 / 3$ & $1 / 5$ \\
Highest & 7000 & N/A & 0 & 0
\end{tabular}


Table III. Enumeration of strategies for the two-stage decision problem

\begin{tabular}{c|c|c|c|c}
$j_{h, p}$ & \multicolumn{1}{c}{$h$} & \multicolumn{1}{c}{$\begin{array}{c}\text { Decision } \\
\text { Node 1 }\end{array}$} & \multicolumn{1}{c}{$\begin{array}{c}\text { Decision } \\
\text { Node 2 }\end{array}$} \\
\hline 1 & & $\mathrm{Y}$ & 1 & $\mathrm{~N}$ \\
2 & 1 & 2 & $\mathrm{Y}$ \\
\cline { 2 - 5 } 3 & \multirow{2}{*}{2} & $\mathrm{~N}$ & 1 & $\mathrm{~N}$ \\
4 & & & 2 & $\mathrm{Y}$ \\
\hline
\end{tabular}


Table IV. Summary of reference transition scenario input parameters.

\begin{tabular}{clccc}
\multicolumn{1}{c}{ Year } & \multicolumn{1}{c}{ Value } & Unit & Reactor Type \\
\hline A. Simulation Parameters & & & \\
\hline 2015 & Start Year & 2015 & $\mathrm{yr}$ & - \\
2100 & Decision-making End Year & 2100 & $\mathrm{yr}$ & - \\
2160 & Simulation End Year & 2160 & $\mathrm{yr}$ & - \\
2015 & Legacy SNF Stockpile & 0.0 & $\mathrm{tIHM}$ & - \\
2015 & Electricity Demand Growth Rate & 1.25 & $\% / \mathrm{yr}$ & - \\
\hline B. Reactor & Fleet Data & & & \\
\hline 2015 & Initial Generation Capacity & 100 & $\mathrm{GW}$ & - \\
2015 & Initial Capacity Data & 100 & $\%$ & APWR \\
- & New Reactor Lifetime & 60 & $\mathrm{yr}$ & - \\
\hline & Reactor Construction Time & 4 & $\mathrm{yr}$ & -
\end{tabular}


Table V. Reactor input parameters.

A. Reactor power parameters

\begin{tabular}{|c|c|c|c|}
\hline \multicolumn{4}{|c|}{ Value (Reactor Type) } \\
\hline & $(\mathbf{A P W R})^{\mathrm{b}}$ & $(\mathbf{F R})^{\mathrm{a}}$ & \\
\hline Plant Size & 1450 & 380 & $\mathrm{MW}_{\mathrm{e}}$ \\
\hline Thermal Efficiency & 0.34 & 0.38 & $\frac{\mathrm{MW}_{e}}{\mathrm{MW}_{t}}$ \\
\hline Recirculating Power & 0.02 & 0.02 & $\frac{\mathrm{MW}_{\mathrm{e}} \text { recycled to plant systems }}{\mathrm{MW}_{\mathrm{e}} \text { produced }}$ \\
\hline Discharge Burnup & 60 & 134 & $\frac{\mathrm{MWd}_{t}}{\mathrm{~kg} \mathrm{IHM}^{\prime}}$ \\
\hline Availability & 0.90 & 0.82 & Load factor \\
\hline Enrichment & 4.9 & 0.25 & $\%{ }^{235} \mathrm{U}$ \\
\hline
\end{tabular}

\section{B. Reactor fuel recipes}

\section{Value (Reactor Type)}

\begin{tabular}{ccc} 
& $(\mathbf{A P W R})$ & $(\mathbf{F R})$ \\
\cline { 2 - 3 }$x_{U}$ & 1 & 0.6661 \\
$x_{P u}$ & 0 & 0.29571 \\
$x_{M A}$ & 0 & 0.03819 \\
$y_{U}$ & 0.92446 & 0.58709 \\
$y_{P u}$ & 0.01242 & 0.23899 \\
$y_{M A}$ & 0.00129 & 0.03144
\end{tabular}

${ }^{\mathrm{a}}$ From Appendix $\mathrm{C}$ in Dixon et $\mathrm{al}^{24}$.

${ }^{\mathrm{b}}$ From NEA ${ }^{27}$.

${ }^{\mathrm{c}}$ Fuel recipe from VISION input files; FR fuel recipe taken at equilibrium. 
Table VI. Unit cost data for reference scenario

\begin{tabular}{|c|c|c|c|}
\hline Technology & Value & Unit & Source \\
\hline Uranium Mining \& Milling & 75 & $\$ / \mathrm{kg} \mathrm{U}$ as $\mathrm{U}_{3} \mathrm{O}_{8}$ & Table A1-22 \\
\hline Conversion to $\mathrm{UF}_{6}$ & 10 & $\$ / \mathrm{kg} \mathrm{U}$ as $\mathrm{U}_{3} \mathrm{O}_{8}$ & Table B-2 \\
\hline Enrichment & 110 & $\$ /$ SWU & Table C1-5 \\
\hline Fuel Fabrication & 250 & $\$ / \mathrm{kg}$ IHM & Table D1-1-3 (for APWR) \\
\hline Transportation to Reactor & 34 & $\$ / \mathrm{kg}$ IHM & Table O2-8 \\
\hline SNF Storage & 300 & $\$ / \mathrm{kg}$ IHM & Table E1-4 \\
\hline SNF Transportation & 92 & $\$ / \mathrm{kg}$ IHM & Table O1-7 \\
\hline Reprocessing $^{\mathrm{a}}$ & 1370 & $\$ / \mathrm{kg}$ IHM & Table F1-4 (Co-Ex) \\
\hline SNF Disposal & 1000 & $\$ / \mathrm{kg}$ IHM & Table L-7 \\
\hline HLW Disposal & 4000 & $\$ / \mathrm{kg}$ FP and MA in HLW & Table L-8 \\
\hline Separated Actinide Storage & 440 & $\$ / \mathrm{kg} / \mathrm{yr}$ & Table E3-7 \\
\hline $\begin{array}{l}\text { Separated Actinide Vitrification \& } \\
\text { Disposal }\end{array}$ & 5000 & $\$ / \mathrm{kg}$ FP & Table G-1 \\
\hline
\end{tabular}

${ }^{\text {a }}$ Includes HLW storage and vitrification 
Table VII. Perfect information strategies for end-state histories

\begin{tabular}{|c|c|c|c|c|c|c|c|}
\hline $\begin{array}{l}\text { Build in } \\
2030 ?\end{array}$ & $\begin{array}{c}\text { FR cost 2030- } \\
45\end{array}$ & $\begin{array}{c}\text { FR cost } 2045- \\
60\end{array}$ & $\begin{array}{l}\text { Build in } \\
2045 ?\end{array}$ & $\begin{array}{c}\text { FR cost 2060- } \\
\text { onward }\end{array}$ & $\begin{array}{l}\text { Build in } \\
2060 ?\end{array}$ & $\begin{array}{l}\text { Build in } \\
2075 ?\end{array}$ & $\begin{array}{l}\text { Integrated } \\
\text { Cost }[\$ T]\end{array}$ \\
\hline $\mathrm{Y}$ & \multirow{3}{*}{ Low + FOAK } & \multirow{3}{*}{ Low } & $\bar{Y}$ & Lowest & $\bar{Y}$ & N/A & 11.497 \\
\hline Y & & & Y & Low & Y & N/A & 11.566 \\
\hline $\mathrm{Y}$ & & & $\mathrm{Y}$ & Medium & $\mathrm{N}$ & N/A & 11.615 \\
\hline $\mathrm{Y}$ & \multirow{3}{*}{$\begin{array}{c}\text { Medium + } \\
\text { FOAK }\end{array}$} & \multirow{3}{*}{ Medium } & $\mathrm{Y}$ & Low & $\mathrm{Y}$ & N/A & 11.710 \\
\hline Y & & & Y & Medium & $\mathrm{N}$ & N/A & 11.757 \\
\hline $\mathrm{Y}$ & & & $\mathrm{Y}$ & High & $\mathrm{N}$ & N/A & 11.757 \\
\hline $\mathrm{N}$ & \multirow{3}{*}{ High + FOAK } & \multirow{3}{*}{ High } & $\mathrm{N}$ & Medium & $\mathrm{N}$ & N/A & 11.765 \\
\hline $\mathrm{N}$ & & & $\mathrm{N}$ & High & $\mathrm{N}$ & N/A & 11.765 \\
\hline $\mathrm{N}$ & & & $\mathrm{N}$ & Highest & $\mathrm{N}$ & N/A & 11.765 \\
\hline
\end{tabular}

"Y" - build reprocessing facility; "N" - don't build reprocessing facility; "W" - wait; "N/A" - not applicable 


\section{FIGURE CAPTIONS}

Fig. 1. Reference closed fuel cycle.

Fig. 2. Reference transition scenario.

Fig. 3. Example two-stage decision problem.

Fig. 4. Optimal hedging strategies and associated regrets for reference transition scenario.

Fig. 5. Expected regret for stage 1 hedging strategies given probability of high intermediate state occurring.

Fig. 6. Expected regret for stage 2 hedging strategies given medium intermediate state occurred and probability of high end-state occurring. 


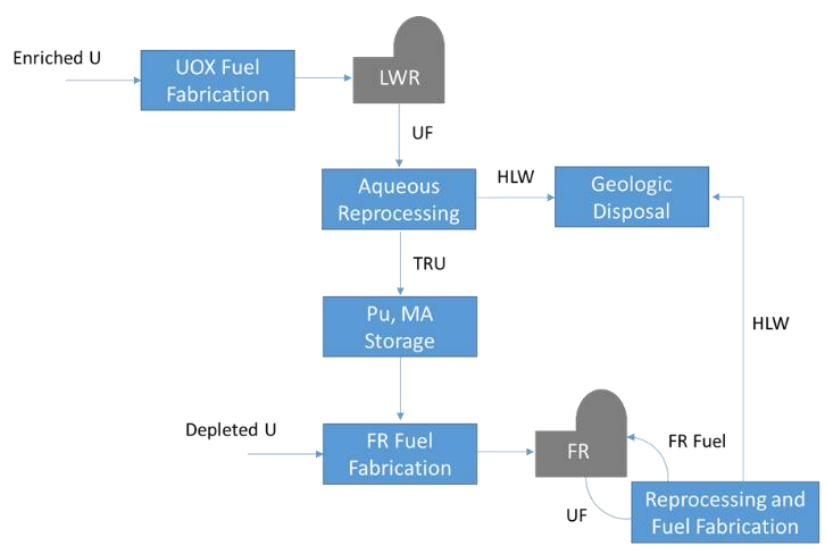

Fig. 1. Reference closed fuel cycle. 


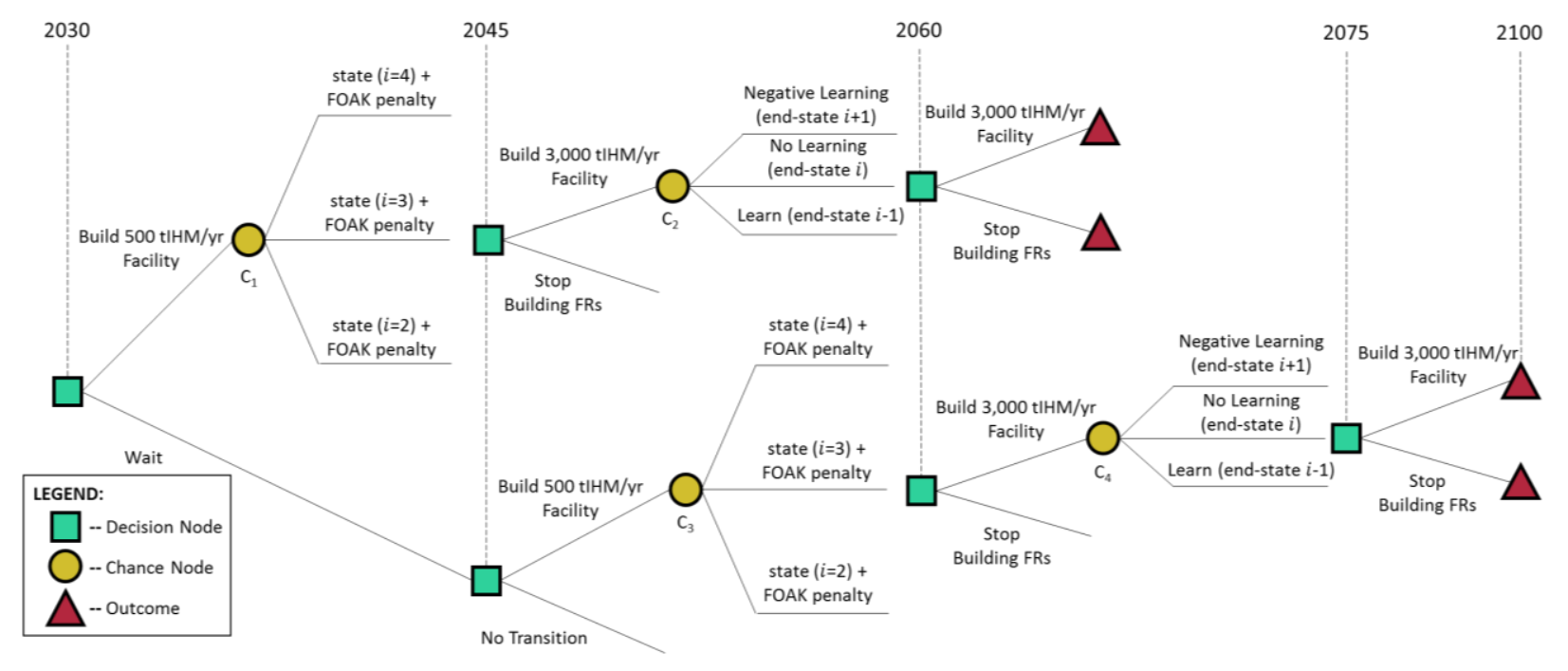

Fig. 2. Reference transition scenario 


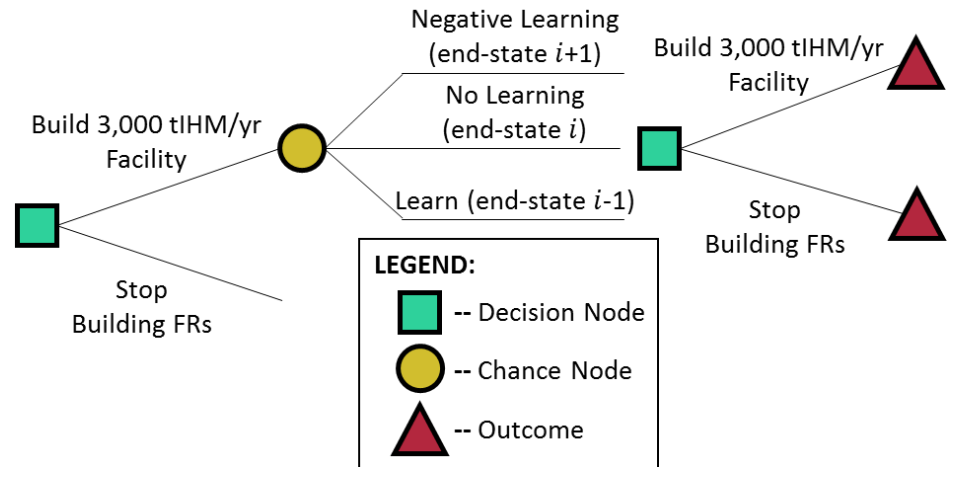

Fig. 3. Example two-stage decision problem. 


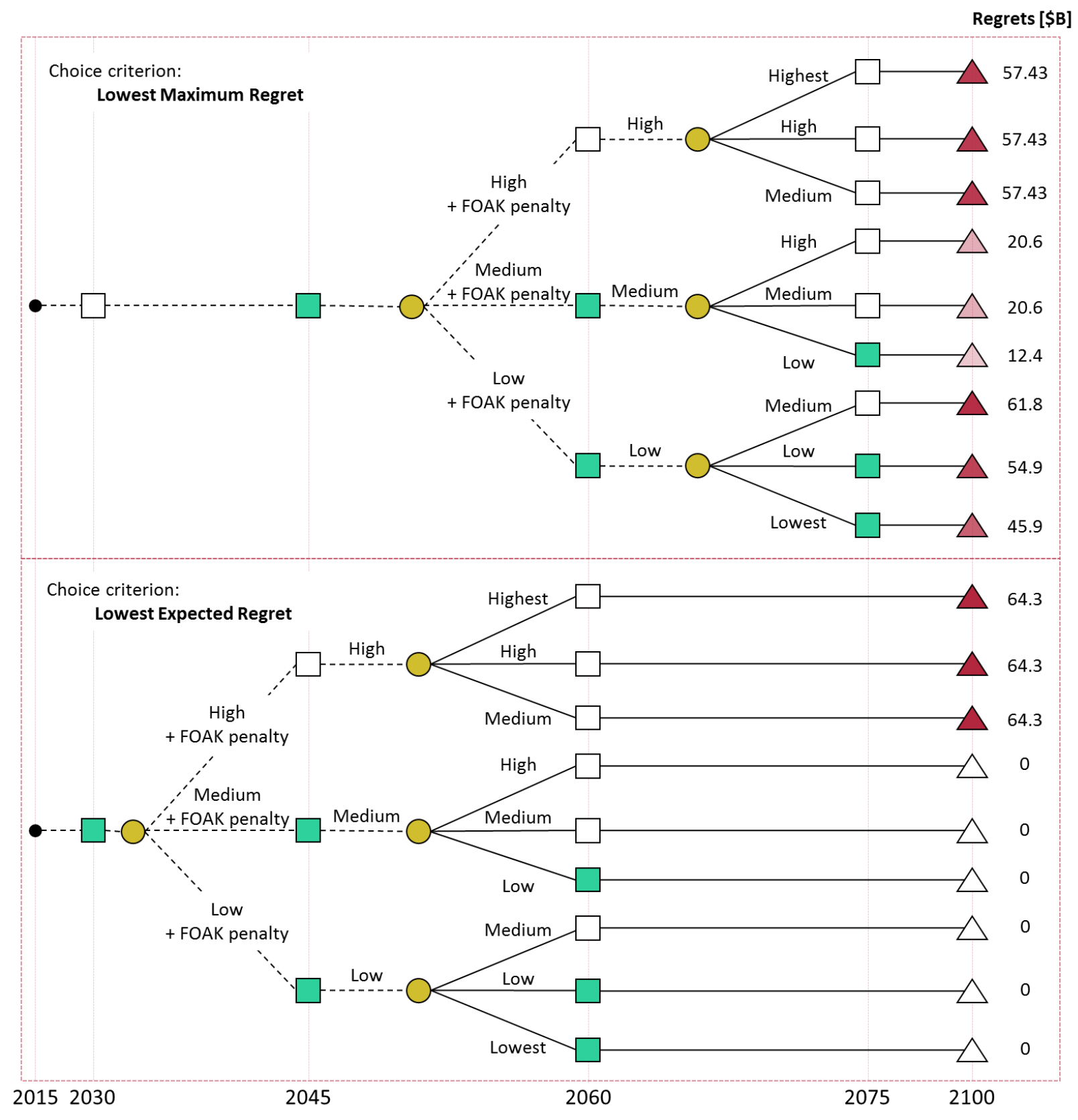

Fig. 4. Optimal hedging strategies and associated regrets for reference transition scenario. 


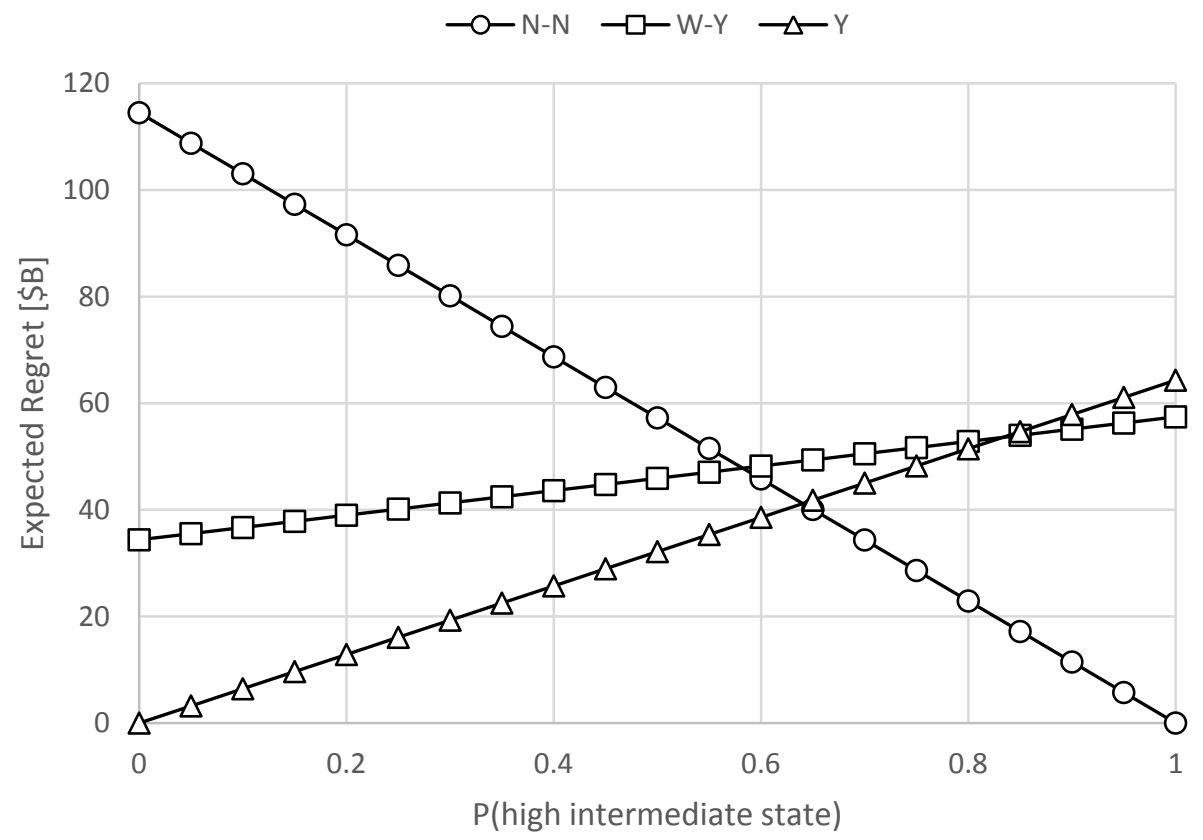

Fig. 5. Expected regret for stage 1 hedging strategies given probability of high intermediate state occurring. 


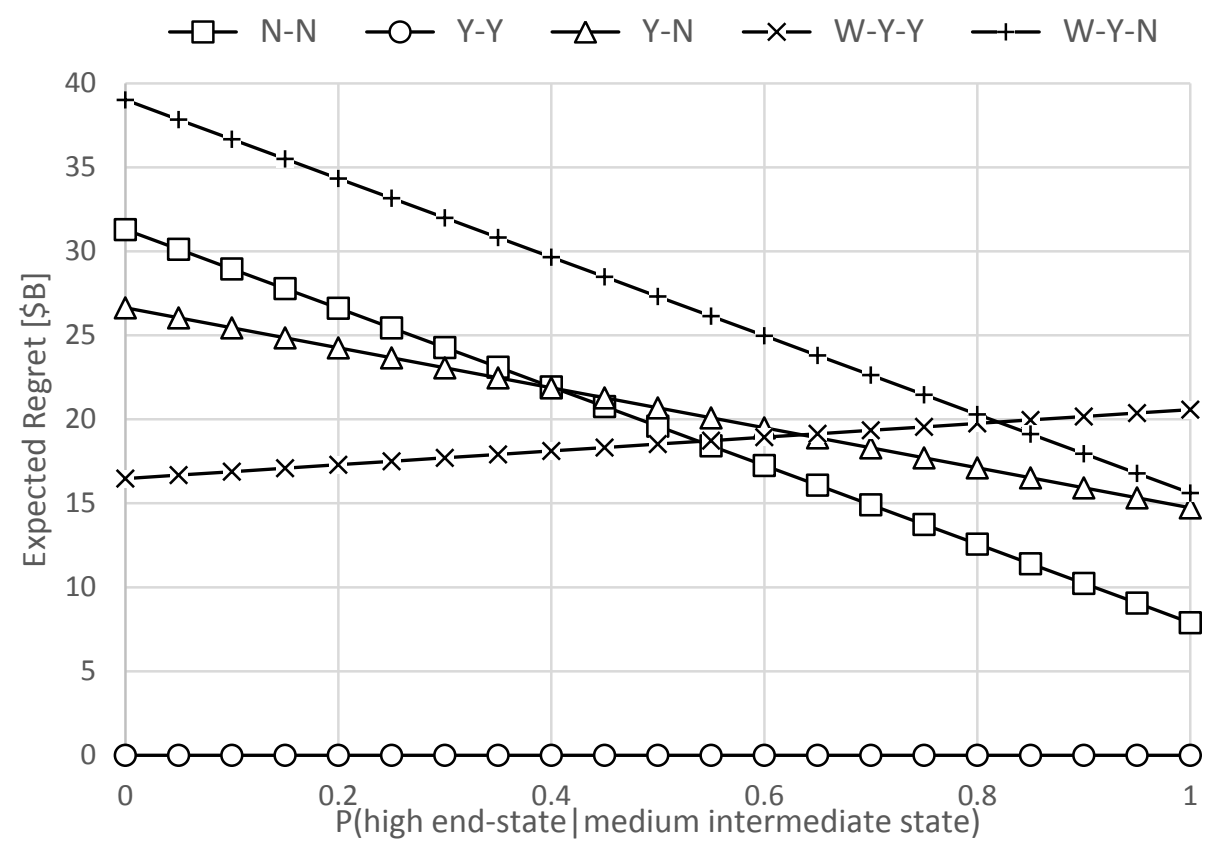

Fig. 6. Expected regret for stage 2 hedging strategies given medium intermediate state occurred and probability of high end-state occurring. 